\title{
PARANEOPLASTIC RHEUMATIC DISEASES: 3 CASES AND REVIEW OF LITERATURE
}

Diego Riveros Logrado 1, , Sérgio Mameri Meireles de Souza', Silvio dos Reis Junior', Luiz Felipe Adsuara de Sousa', Dawton Yukito Torigoe ${ }^{1}$

1. Faculdade de Ciências Médicas da Santa Casa de São Paulo, São Paulo (SP), Brazil.

*Corresponding author: dilogrado@gmail.com

\section{BACKGROUND}

Systemic sclerosis (SSc) is characterized by autoimmune inflammation, vascular damage and extensive fibrosis, which is started around 30 to 50 years old, being uncommon before 25 years old. Patients with SSc are twice as likely to have cancer. Lung cancer as well as esophageal and oropharyngeal carcinoma are the cancer more associated with SSc. Autoimmune inflammatory myopathies, which form a group of diseases characterized by proximal skeletal muscle weakness, serum elevation of muscle enzymes and may be associated with skin manifestations, such as Gottron's papules and heliotrope in dermatomyositis. The peak incidence of dermatomyositis and polymyositis are, respectively, 60 to 69 years old and 50 to 59 years old. Among the malignant neoplasms most frequently associated with inflammatory myopathies in women are breast and ovarian neoplasms. The relative risk of neoplasia in dermatomyositis varies from 3 to 12.6, being higher in the first 3 to 5 years of the disease. The objective is to correlate neoplasms as triggers of systemic autoimmune rheumatic diseases (SARD).

\section{CASE REPORT}

We present three patients with SARD which developed a diagnosis of neoplasms later. The first case refers to a 19-year-old woman complaining that at 17 years of age, who started pelvic and scapular girdle weakness, arthralgia and high dysphagia, being later diagnosed with polymyositis. During the investigation to rule out neoplasm, she was diagnosed with ovarian teratoma. The second case concerns a female patient who started at the age of 47 years old the symptoms of pelvic girdle weakness, arthralgia, prolonged morning stiffness, Gottron's papules, heliotrope and diffuse skin rash, receiving the diagnosis of breast cancer in the following years. A 49-year-old female patient, representing the third case, with a three-year history of scapular girdle weakness, arthralgia with prolonged morning stiffness, Gottron's papules, telangiectasias, skin thickening and dysphagia, later diagnosed with a SSc triggered by mucinous adenocarcinoma of the appendix with bilateral adnexal metastasis, submitted to surgical procedure, subsequently evolving to death. The summary of symptoms is shown in Fig. 1.

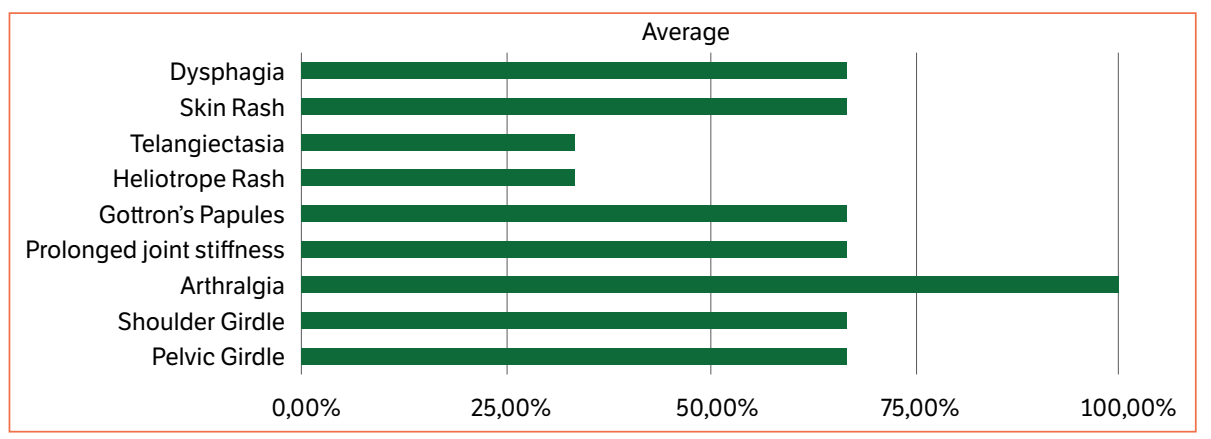

Figure 1. Symptons.

\section{CONCLUSION}

The cases 1 and 2 are in accordance with the literature evidencing the correlation between gynecological neoplasms and autoimmune inflammatory myopathies. About the case 3, although esophageal adenocarcinoma is the most related to SSc, it is clear that this same histological type of cancer is also correlated with other sites, such as the appendix. 